\title{
Uso de instrumentos assistenciais pelo enfermeiro no rastreio de sintomas
}

\section{depressivos em idosos}

\author{
Use of care tools by nurses to screen for depressive symptoms in the elderly \\ Uso de herramientas de atención por parte de las enfermeras para detectar síntomas depresivos en
}

los ancianos

Recebido: 09/12/2021 | Revisado: 20/12/2021 | Aceito: 21/12/2021 | Publicado: 03/01/2022

\author{
Daniela Silva Marques \\ ORCID: https://orcid.org/0000-0003-1706-2200 \\ Universidade Federal do Maranhão, Brasil \\ E-mail: daniela.marqueslv@gmail.com \\ Keyla Cristina Nogueira Durans \\ ORCID: https://orcid.org/0000-0003-4963-5448 \\ Universidade Federal do Maranhão, Brasil \\ E-mail: Keyla.durans@discente.ufma.br \\ Erica Amador de Cristo \\ ORCID: https://orcid.org/0000-0002-1627-9275 \\ Universidade Federal do Maranhão, Brasil \\ E-mail: ericaamador13@gmail.com \\ Julyana Suelen Rodrigues Fonseca \\ ORCID: https://orcid.org/0000-0002-7777-7681 \\ Universidade Federal do Maranhão, Brasil \\ E-mail: Suelenfonseca.jf@gmail.com \\ Adryemerson Pena Forte Ferreira \\ ORCID: https://orcid.org/0000-0001-6423-9756 \\ Universidade Federal do Maranhão, Brasil \\ E-mail: Adryemerson.pena@gmail.com \\ João de Deus Cabral Junior \\ ORCID: https://orcid.org/0000-0003-2339-9635 \\ Universidade do Estado do Rio de Janeiro, Brasil \\ Universidade Federal do Maranhão, Brasil \\ E-mail: joao.dcj@ufma.br
}

\begin{abstract}
Resumo
Objetivo: caracterizar por meio da literatura nacional, os instrumentos assistenciais utilizados pelo enfermeiro na identificação de sintomas depressivos na população idosa atendida. Método: Revisão Integrativa de Literatura que buscou artigos nacionais escritos entre os anos de 2015-2021 que tratassem sobre as ferramentas usadas pelo Enfermeiro para auxílio no rastreio da depressão senil. Os manuscritos foram filtrados nas seguintes plataformas: Scientific Eletronic Library Online (SCIELO), Literatura Latino-Americana e do Caribe em Ciências da Saúde (LILACS) e Biblioteca Virtual em Saúde (BVS), utilizando cruzamentos com os seguintes descritores disponíveis no site Descritores em Ciência da Saúde (DeCS): Depressão, Idoso, Enfermagem e Enfermagem Geriátrica. Resultados: Ao todo, foram selecionados para a pesquisa 15 artigos, a maior parte do ano de 2020. A Escala de Depressão Geriátrica foi o principal instrumento de escolha do enfermeiro, aliado ao processo de anamnese. A partir dos resultados foram elencadas três categorias de discussão: "principais escalas para rastreio da depressão em idosos", "o envelhecer saudável e a depressão: solidão e resiliência na terceira idade" e "condutas do enfermeiro mediante o rastreio da depressão". Considerações Finais: Os enfermeiros utilizaram-se de abordagens multidimensionais, que permitiram a avaliação de outros parâmetros importantes a qualidade de vida do idoso além do estado depressivo, porém chama-se a atenção à necessidade de criação de novos instrumentos direcionados ao objeto da Enfermagem e maior apropriação do PE, permitindo priorizar o cuidado centrado na pessoa e suas necessidades, otimizando o cuidado e assistência.
\end{abstract}

Palavras-chave: Depressão; Idoso; Enfermagem; Enfermagem geriátrica.

\begin{abstract}
Objective: to characterize, through the national literature, the care tools used by nurses to identify depressive symptoms in the elderly population they care for. Method: Integrative Literature Review that searched for national articles written between the years 2015-2021 that dealt with the tools used by nurses to assist in the screening of senile depression. The manuscripts were filtered in the following platforms: Scientific Eletronic Library Online (SCIELO), Latin American and Caribbean Literature on Health Sciences (LILACS) and Virtual Health Library (BVS), using cross-references with
\end{abstract}


the following descriptors available on the Health Science Descriptors (DeCS) website: Depression, Elderly, Nursing and Geriatric Nursing. Results: A total of 15 articles were selected for the search, mostly from the year 2020. The Geriatric Depression Scale was the main instrument of choice for nurses, together with the anamnesis process. From the results, three categories of discussion were listed: "main scales for screening depression in the elderly", "healthy aging and depression: loneliness and resilience in old age", and "nurses' actions when screening for depression". Final Considerations: The nurses used multidimensionais approaches, which allowed for the assessment of other important parameters for the quality of life of the elderly in addition to the depressive state. However, attention is drawn to the need to create new instruments directed at the object of Nursing and a greater appropriation of the NP, allowing for the prioritization of care centered on the person and his/her needs, optimizing care and assistance.

Keywords: Depression; Elderly; Nursing; Geriatric nursing.

\section{Resumen}

Objetivo: caracterizar, a través de la literatura nacional, los instrumentos de asistencia utilizados por el enfermero en la identificación de los síntomas de depresión en la población atendida. Método: Revisión bibliográfica integradora en la que se buscaron artículos nacionales escritos entre los años 2015-2021 que trataran sobre las herramientas utilizadas por la Enfermería para ayudar en el cribado de la depresión senil. Los manuscritos fueron filtrados en las siguientes plataformas: Scientific Eletronic Library Online (SCIELO), Latin American and Caribbean Literature on Health Sciences (LILACS) y Virtual Health Library (VHL), utilizando referencias cruzadas con los siguientes descriptores disponibles en el sitio web de Health Science Descriptors (DeCS): Depression, Elderly, Nursing y Geriatric Nursing. Resultados: Se seleccionaron un total de 15 artículos para la búsqueda, la mayoría del año 2020. La Escala de Depresión Geriátrica fue el principal instrumento de elección del enfermero, junto con el proceso de anamnesis. A partir de los resultados se establecieron tres categorías de discusión: "principales escalas de rastreo de la depresión en los individuos", "el nivel de salud y la depresión: solidez y resiliencia en la tercera edad" y "las consecuencias de la enfermedad en el rastreo de la depresión". Consideraciones Finacionales: Los enfermeros utilizaron enfoques multidimensionales, que permitieron evaluar otros parámetros importantes para la calidad de vida del individuo además del estado depresivo, por lo que se prestó atención a la necesidad de crear nuevos instrumentos dirigidos al objetivo de la Enfermería y a una mayor apropiación del PE, permitiendo priorizar el cuidado centrado en la persona y sus necesidades, optimizando el cuidado y la asistencia.

Palabras clave: Depresión; Envejecido; Enfermagem; Enfermagem geriatrica.

\section{Introdução}

A depressão é uma síndrome psiquiátrica altamente prevalente na população em geral, particularizada por alterações psicopatológicas diversas que podem diferenciar-se em relação à sintomatologia, gravidade, curso e prognóstico. Constantemente é caracterizada pela presença de humor predominantemente depressivo, e/ou irritável e diminuição da capacidade de sentir prazer ou alegria que podem estar seguidos de uma sensação subjetiva de cansaço e/ou fadiga, acompanhados de alterações do sono e apetite, desinteresse, pessimismo, lentidão e ideias de fracasso (Lima et al., 2016).

De acordo com a Organização Mundial de Saúde (OMS) a depressão é considerada um preocupante problema de saúde pública e estima-se que 154 milhões de pessoas sejam acometidos em todo o mundo, sendo vista como a quarta causa específica de incapacitação social, com perspectiva de ser a segunda causa em países desenvolvidos e a primeira nos países em desenvolvimento aproximadamente até o ano de 2020. Estima-se que há uma predominância entre os idosos com o percentual de $15 \%$ de prevalência para algum sintoma depressivo (Guimarães et al., 2019).

$\mathrm{Na}$ atualidade, evidencia-se o aumento do quantitativo de pessoas com 65 anos ou mais nos países em desenvolvimento. No Brasil, a representatividade deste público é de 14,5 milhões, equivalente a 8,6\% do total da população do país, com projeção para 9,7\% em 2050 (Lima et al., 2016). A OMS, indica como as principais doenças que acometem os idosos os distúrbios cardiovasculares, Hipertensão Arterial Sistêmica, Acidente Vascular Encefálico, Diabetes tipo II, Doença Pulmonar Obstrutiva Crônica, doenças musculoesqueléticas, e grande ênfase aos distúrbios mentais (Cardoso et al., 2018).

A população idosa apresenta maior vulnerabilidade para problemas mentais, com destaque para a depressão, em vista às percepções das perdas funcionais e sociais que a senescência conduz consigo. As limitações vivenciadas no processo de envelhecimento muitas vezes não são compreendidas pelos idosos e até mesmo por pessoas de sua convivência. A ausência do enfrentamento adequado dessas condições é capaz de levar os mesmos a desenvolverem sentimento de impotência e 
incapacidade, facilitando assim o surgimento da depressão (Costa et al., 2017).

Tendo em vista a estigmatização social, associada aos sintomas causados pela depressão, alguns idosos julgam ser inútil expor o problema ao profissional de saúde. Simultaneamente a isso, se os profissionais de saúde não estiverem atentos para a identificação desses sinais e sintomas ou os considerarem parte normal do processo de envelhecimento, seguramente o diagnóstico passará despercebido. Além das evidências, a depressão constantemente é desconsiderada por profissionais de saúde e, mais importante, não é tratada. Estima-se que 50\% dos idosos depressivos não são diagnosticados pelos profissionais assistenciais que exercem atividade na atenção primária, devido alguns dos sintomas serem semelhantes ao processo natural do envelhecimento (Ramos et al., 2019).

Mediante estes dados e a agenda de prioridades de pesquisa do Ministério da Saúde, que inclui a saúde do idoso como um dos pilares principais, o profissional enfermeiro tem espaço para ampliar seu conhecimento e ações de cuidados em dois âmbitos: por meio da pesquisa científica, que faz reconhecer e divulgar as dificuldades do público idoso e assim pôr em pauta suas necessidades, e na área assistencial, ao ponto que acompanha este indivíduo também em seu estágio avançado de vida. O subdiagnóstico de uma doença como a depressão tem um impacto importante nas relações interpessoais do idoso e no controle de outras doenças que podem aparecer em decorrência dela, levando em casos mais extremos, ao suicídio (Uchoa et al., 2019).

Neste contexto, questiona-se: "quais os instrumentos assistenciais que podem ser utilizados pelo enfermeiro no rastreio de sintomas depressivos em idosos?", observado que o uso de instrumentos para detecção precoce de sintomas depressivos permite a estratificação de risco e prevenção do agravamento de condições adversas vividas pelo idoso (Wanderley et al., 2019). Deste modo, o objetivo deste escrito foi caracterizar por meio da literatura nacional, os instrumentos assistenciais utilizados pelo enfermeiro na identificação de sintomas depressivos na população idosa atendida.

\section{Metodologia}

Este estudo tem cunho exploratório e foi realizado por meio da Revisão Integrativa de literatura (RI). A RI é uma metodologia de pesquisa que objetiva analisar e sistematizar as publicações científicas relacionadas a determinado tema. Constitui-se como importante ferramenta dentro dos estudos que compreendem a área da saúde, uma vez que estimula a capacidade de síntese de ideias e de avaliação crítica de achados na literatura, culminando na criação de meios de comparação dos materiais disponíveis e por consequência gerando mais conhecimento dentro do tema estudado, visto que evidencia possíveis fragilidades e potencialidades dentro do assunto (Sousa et al., 2018).

Segundo Sousa e colaboradores (2018), a revisão se estabelece como modo de produção pertinente em estudos de Enfermagem, e ainda sobre este, os autores elencam 6 etapas que devem ser seguidas para uma escrita adequada da revisão de literatura. As mesmas conduziram este método e são: 1) identificação do tema e seleção da questão de pesquisa para a elaboração da revisão integrativa; 2) estabelecimento de critérios para inclusão e exclusão de estudos ou pesquisa de literatura; 3) definição das informações a serem extraídas dos estudos selecionados/categorização dos estudos; 4) avaliação dos estudos incluídos na revisão integrativa; 5) interpretação dos resultados e, 6) apresentação da revisão/síntese do conhecimento.

A questão norteadora desta pesquisa, trata sobre "Quais os instrumentos assistenciais que podem ser utilizados pelo enfermeiro no rastreio de sintomas depressivos em idosos?". A pergunta foi elaborada através da estratégia proposta pelo acrônimo População Interesse Contexto - PICo, onde os autores compreenderam como População: Idosos com sinais e sintomas depressivos, Interesse: Conhecimento dos instrumentos assistenciais utilizados e Contexto: durante a prática do Enfermeiro.

Para responder ao questionamento, realizou-se primeira etapa através de uma pesquisa eletrônica, utilizando as seguintes bases de dados: Scientific Eletronic Library Online (SCIELO), Literatura Latino-Americana e do Caribe em Ciências da Saúde (LILACS) e Biblioteca Virtual em Saúde (BVS), utilizando cruzamentos com os seguintes descritores disponíveis no site Descritores em Ciência da Saúde (DeCS): Depressão, Idoso, Enfermagem e Enfermagem Geriátrica. 
Como critérios de inclusão, foram selecionados todos os artigos que estavam em português, disponíveis na íntegra pela base de dados, disponíveis entre os anos de 2015-2020, e que abordaram a temática principal do estudo. Foram excluídos os trabalhos sem disponibilidade para leitura completa, que não estavam no idioma citado anteriormente, que fossem revisões de literatura, teses ou dissertações, assim como artigos repetidos e que não tinham aproximação com o objeto estudado. Para otimizar o processo de visualização, os autores utilizaram o fluxograma proposto pelo Preferred Reporting of Systematic Reviews and Meta-Analyses - PRISMA (Figura 1).

Figura 1 - Fluxograma de artigos selecionados conforme estratégia PRISMA. Pinheiro, Maranhão, 2021.

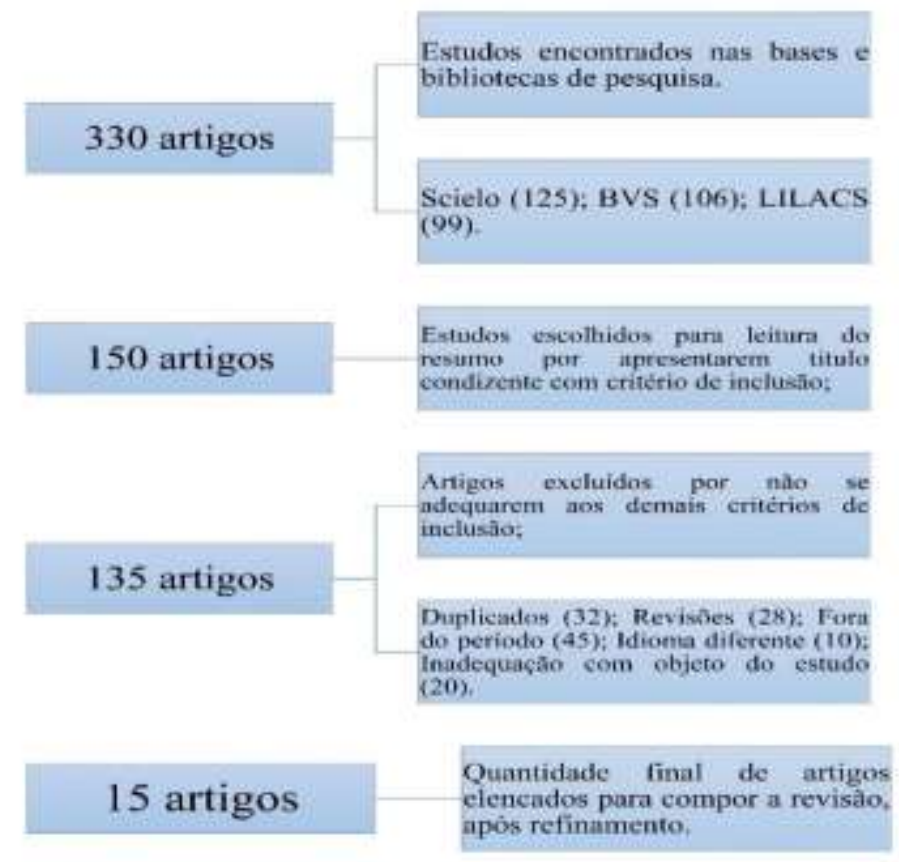

Fonte: Autores (2021).

Após a primeira seleção de estudos, foi realizada leitura minuciosa em busca daqueles que trabalharam sobre as ferramentas assistenciais que podem ser utilizadas na prática de enfermagem, sejam elas escalas, entrevistas, cartilhas ou outros métodos de rastreamento de sintomatologia depressiva entre os idosos. Mediante isto, os artigos selecionados foram organizados em quadro-síntese que teve a seguinte sequência: Ano, título, autores, periódico da publicação com nível de evidência da pesquisa e resultados. Para caracterização do nível de evidência científica de cada artigo foi utilizado o método proposto por Stillwell et al. (2010), exposto pela Figura 2. 
Figura 2 - Nível de evidência utilizada para os estudos sobre depressão senil. Pinheiro, Maranhão, 2021.

\begin{tabular}{cl}
\hline nível I & Metanálise de estudos controlados e randomizados \\
\hline nível II & Estudo experimental \\
\hline nível III & Estudo quase experimental \\
\hline nível IV & Estudo descritivo/não experimental ou com abordagem qualitativa \\
\hline nível V & Relato de caso ou experiência \\
\hline nível VI & Consenso e opinião de especialistas \\
\hline
\end{tabular}

Fonte: Autores (2021).

O presente estudo, por não estar diretamente relacionado com pesquisa que envolve seres humanos, dispensou a apreciação pelo Comitê de Ética em Pesquisa, como rege a Resolução 466/12 do Conselho Nacional de Saúde. Ainda segundo esta resolução, devido este estudo ser baseado em agregados de dados secundários com acesso livre, dispensou-se a utilização do Termo de Consentimento Livre e Esclarecido (TCLE).

\section{Resultados}

Ao todo, 15 artigos compuseram os resultados da pesquisa, sendo a sua maioria publicada no ano de 2020 (4). As pesquisas estavam indexadas em sua maioria por periódicos referentes a Enfermagem, com destaque a Revista Brasileira de Enfermagem, com duas publicações. Todos os artigos apresentaram nível de evidência IV, por contemplarem em sua metodologia pesquisas descritivas e/ou com avaliação qualitativa. Destaca-se o uso de análises estatísticas simples pelos enfermeiros, visando conferir maior significância aos estudos.

Outro ponto considerável faz jus a abordagem multidimensional proposta pelos enfermeiros nos artigos, permitindo a associação entre os sintomas depressivos e outras condições importantes a este público como a resiliência, capacidade funcional, qualidade de vida, estado nutricional, ocorrência de violência domiciliar e autoestima do idoso (Antequera et al., 2020; Araújo et al., 2017; Didoné et al., 2020).

Para observação e rastreio dos sintomas depressivos, utilizaram-se de ferramentas como: entrevistas dirigidas, questionários de criação própria e escalas validadas a nível internacional, como é disposto pelo Quadro 1. 


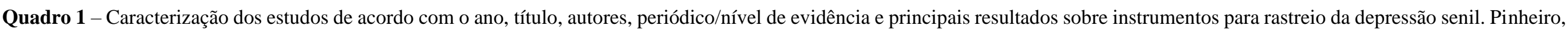
MA, Brasil, 2021.

\begin{tabular}{|c|c|c|c|c|}
\hline Ano & Titulo & Autores & Revista & Principais resultados \\
\hline 2015 & $\begin{array}{l}\text { Prevalência de sintomas depressivos } \\
\text { e sinais de demência em idosos na } \\
\text { comunidade }\end{array}$ & Lentsck et al. & $\begin{array}{l}\text { Rev. Eletrônica de } \\
\text { Enfermagem } \\
\text { NIVEL IV }\end{array}$ & $\begin{array}{l}\text { O estudo foi realizado por meio de inquérito domiciliar e utilizou as ferramentas Brazil Old Age Schedule (BOAS) e a escala Short- } \\
\text { Care para rastreio dos sintomas depressivos em idosos. Para comprovar a associação entre os resultados e variáveis } \\
\text { sociodemográficas, utilizou-se de análises estatísticas. Algumas informações divergentes foram encontradas nas falas dos idosos, } \\
\text { como a falta de certeza entre estar feliz ou triste no momento da pergunta. Os sintomas depressivos estiveram associados ao sexo } \\
\text { feminino e a morar sozinho. }\end{array}$ \\
\hline 2015 & $\begin{array}{l}\text { Depressão e estado nutricional de } \\
\text { idosos participantes do Programa } \\
\text { Hiperdia }\end{array}$ & Pereira et al. & $\begin{array}{l}\text { Rev Rene. } \\
\text { NIVEL IV }\end{array}$ & $\begin{array}{l}\text { Utilizou-se a Escala de Depressão Geriátrica com } 15 \text { itens, associada a mensuração de parâmetros corporais. Os autores não } \\
\text { encontraram relação significativa entre o perfil nutricional dos participantes e a ocorrência de depressão, porém, observou-se maior } \\
\text { proporção de idosos com depressão mínima ou moderada que tinham maior risco de desenvolvimento de doenças cardiovasculares } \\
(61,8 \%) \text { e, os idosos com sobrepeso que apresentavam depressão mínima ou moderada }(67,6 \%) \text {. }\end{array}$ \\
\hline 2016 & $\begin{array}{l}\text { Conhecimento dos idosos sobre os } \\
\text { sinais e sintomas da depressão }\end{array}$ & $\begin{array}{l}\text { Andrade; } \\
\text { Ferreira; } \\
\text { Aguiar. }\end{array}$ & $\begin{array}{l}\text { Saúde em Redes } \\
\text { NIVEL IV }\end{array}$ & $\begin{array}{l}\text { Para o desenvolvimento da pesquisa, foi feita uma adaptação da Escala de Depressão Geriátrica de Yesavage (GDS) pelos autores, } \\
\text { indagando sobre aquilo que os idosos acreditam ser sintomas de depressão. Aos resultados foi possível constatar que apesar de } \\
88 \% \text { deles nunca terem obtido diagnóstico para a doença, } 66 \% \text { conhecem ou convivem com alguém que a apresenta. A maior parte } \\
\text { dos idosos ( } 42 \% \text { ) apresentou conhecimento intermediário sobre a depressão, porém, apenas a relacionando com tristeza e solidão, } \\
\text { não tendo conhecimento adequado sobre os demais sinais e sintomas. }\end{array}$ \\
\hline 2017 & $\begin{array}{l}\text { Capacidade funcional e depressão } \\
\text { em idosos }\end{array}$ & Araújo et al. & $\begin{array}{l}\text { Revista de Enfermagem } \\
\text { UFPE } \\
\text { NIVEL IV }\end{array}$ & $\begin{array}{l}\text { As ferramentas utilizadas neste caso foram o Mini Exame do Estado Mental e a Escala de Depressão Geriátrica. Após estatística } \\
\text { descritiva e inferencial feita pelos autores, foi possível constatar que a presença de depressão, rastreada por meio dos testes, tinha } \\
\text { influência negativa no desenvolvimento de atividades avançadas necessárias à vida diária. }\end{array}$ \\
\hline 2017 & $\begin{array}{l}\text { Perfil sociodemográfico, aspectos } \\
\text { familiares, percepção de saúde, } \\
\text { capacidade funcional e depressão } \\
\text { em idosos institucionalizados no } \\
\text { Litoral Norte do Rio Grande do Sul. }\end{array}$ & Güths et al. & $\begin{array}{c}\text { Rev. Bras. Geriatr. Gerontol. } \\
\text { NIVEL IV }\end{array}$ & $\begin{array}{l}\text { Utilizando a Escala de Depressão Geriátrica, os pesquisadores puderam evidenciar } 53 \% \text { dos idosos estudados com sintomas } \\
\text { moderados de depressão, e um deles com sintomas severos. Um fato importante relatado trata sobre a instituição não possuir } \\
\text { nenhum profissional de nível superior trabalhando com os idosos. }\end{array}$ \\
\hline 2018 & $\begin{array}{l}\text { Fragilidade, sintomas depressivos e } \\
\text { Qualidade de vida: um estudo com } \\
\quad \text { idosos institucionalizados }\end{array}$ & Melo et al. & $\begin{array}{l}\text { Rev baiana enferm. } \\
\text { NIVEL IV }\end{array}$ & $\begin{array}{l}\text { A Escala de Depressão Geriátrica (GDS-15) foi utilizada juntamente aos instrumentos Quality of Life Scales for Nursing Home } \\
\text { Residents (para qualidade de vida) e Indicador de Fragilidade de Tilburg (TFI). Foi demonstrado pelos autores que os idosos com } \\
\text { condição de fragilidade maior estariam mais propensos ao desenvolvimento de depressão, principalmente em seus domínios físico } \\
\text { e psicológico. }\end{array}$ \\
\hline 2019 & $\begin{array}{l}\text { Prevalência de sintomas depressivos } \\
\text { no idoso hospitalizado: estudo } \\
\text { comparativo. }\end{array}$ & Serra et al. & $\begin{array}{l}\text { Revista Enfermagem Uerj. } \\
\text { NIVEL IV }\end{array}$ & $\begin{array}{l}\text { O estudo comparou a avaliação que o enfermeiro realizou ao paciente com potencial para o desenvolvimento de depressão } \\
\text { utilizando dois instrumentos distintos: a Escala de Depressão Geriátrica e instrumento com lista de sintomas depressivos, extraídos } \\
\text { das características definidoras dos diagnósticos de enfermagem. Como resultados, a avaliação do enfermeiro mediante diagnósticos } \\
\text { de enfermagem apresentou-se deficiente na detecção dos sintomas, em relação àquela realizada utilizando a escala. }\end{array}$ \\
\hline 2019 & $\begin{array}{l}\text { Fatores associados a sintomas } \\
\text { depressivos e capacidade funcional } \\
\text { em idosos }\end{array}$ & Uchoa et al. & $\begin{array}{l}\text { Cogitare Enfermagem } \\
\text { NIVEL IV }\end{array}$ & $\begin{array}{l}\text { Os enfermeiros valeram-se da Escala de Depressão Geriátrica e de outros instrumentos para a avaliação da capacidade funcional, } \\
\text { fazendo uso de correlação estatística para descobrimento de relações entre causa e efeito. } 22 \% \text { dos idosos apresentaram alguma } \\
\text { sintomatologia e deste, a maior parte concentrava características em comum, com associação estatísticas como: sedentarismo, } \\
\text { autopercepção ruim de saúde e que não participavam de grupos de idosos. }\end{array}$ \\
\hline 2019 & $\begin{array}{l}\text { Resiliência, qualidade de vida e } \\
\text { sintomas depressivos entre idosos } \\
\text { em tratamento ambulatorial* }\end{array}$ & Lima et al. & $\begin{array}{l}\text { Rev. Latino-Am. } \\
\text { Enfermagem. } \\
\text { NIVEL IV }\end{array}$ & $\begin{array}{l}\text { O instrumento Center for Epidemiologic Survey - Depression foi utilizado para rastreamento dos sintomas depressivos e } \\
\text { possibilitar sua associação com a capacidade de resiliência dos idosos participantes do estudo. Segundo resultados, } 64 \% \text { dos idosos } \\
\text { apresentaram sintomatologia depressiva e a presença iminente da depressão contribuiu para a diminuição da resiliência na } \\
\text { população. }\end{array}$ \\
\hline 2020 & $\begin{array}{l}\text { Autoestima e risco para depressão } \\
\text { em idosos residentes em instituições } \\
\text { de longa permanência }\end{array}$ & Santos et al. & $\begin{array}{l}\text { Rev. Espaço para a Saúde } \\
\text { NIVEL IV }\end{array}$ & $\begin{array}{l}\text { Os profissionais utilizaram-se de instrumentos padronizados para o rastreio dos sintomas, aliados a análises estatísticas. Para } \\
\text { avaliação da autoestima foi utilizado escala de autoestima de Rosenberg e para rastreio da depressão a escala abreviada de Zung. } \\
\text { Como resultados, } 52 \% \text { dos participantes apresentavam autoestima baixa, sobretudo aqueles com menor tempo de estadia, e } 48 \%\end{array}$ \\
\hline
\end{tabular}




\begin{tabular}{|c|c|c|c|c|}
\hline & & & & $\begin{array}{l}\text { apresentavam risco para o desenvolvimento de depressão. Os principais achados evidenciados pelos idosos foram: desesperança, } \\
\text { pouca vontade de desempenhar atividades da vida diária, sentimentos de inutilidade, fracasso e insatisfação geral. }\end{array}$ \\
\hline 2020 & $\begin{array}{l}\text { Avaliação da depressão e da } \\
\text { qualidade de vida de idosos } \\
\text { institucionalizados }\end{array}$ & Andrade $e t$ al. & $\begin{array}{l}\text { Revista Enfermagem Atual } \\
\text { In Derme } \\
\text { NIVEL IV }\end{array}$ & $\begin{array}{l}\text { Desenvolvida em uma Instituição de Longa Permanência para Idosos (ILPI), a pesquisa utilizou os instrumentos Escala de } \\
\text { Depressão Geriátrica - GDS aliada a Escala de Qualidade de Vida (SF-36). A maior parte dos idosos não apresentou sintomas } \\
\text { depressivos, porém, tiveram baixo escore na avaliação da qualidade de vida, o que pode os predispor a solidão e condições de } \\
\text { sofrimento mental/psicológico. Houve correlação negativa entre os sintomas depressivos e uma baixa qualidade de vida. }\end{array}$ \\
\hline 2020 & $\begin{array}{l}\text { Fragilidade, depressão e qualidade } \\
\text { de vida: um estudo com idosos } \\
\text { cuidadores }\end{array}$ & Melo et al. & $\begin{array}{l}\text { Rev Bras Enferm. } \\
\text { NIVEL IV }\end{array}$ & $\begin{array}{l}\text { A EDG foi utilizada em associação a demais escalas que permitiam comparações entre a qualidade de vida dos idosos e sua } \\
\text { fragilidade. Utilizando-se de testes estatísticos para correlação, ficou evidente que a depressão estava associada negativamente à } \\
\text { maior fragilidade e pior qualidade de vida do idoso cuidador. }\end{array}$ \\
\hline 2020 & $\begin{array}{l}\text { Fatores associados a sintomas } \\
\text { depressivos em idosos inseridos em } \\
\text { contexto de vulnerabilidade social }\end{array}$ & Didoné et al. & $\begin{array}{l}\text { Rev Bras Enferm. } \\
\text { NIVEL IV }\end{array}$ & $\begin{array}{l}\text { Para a reunião das características relacionadas à qualidade de vida e demais fatores relacionados com a depressão na terceira idade } \\
\text { foi utilizada a Escala de Depressão Geriátrica e mais outros cinco instrumentos. Como fatores protetores da depressão estavam: } \\
\text { melhor percepção sobre qualidade de vida e recebimento de apoio emocional. Por outro lado, fatores preditores a depressão foram: } \\
\text { residir sozinho, apresentar risco de desnutrição e ser do sexo feminino. }\end{array}$ \\
\hline 2021 & $\begin{array}{l}\text { Importância da identificação do } \\
\text { diagnóstico de enfermagem ao } \\
\text { paciente com depressão senil na } \\
\text { atenção básica. }\end{array}$ & Silva et al. & $\begin{array}{l}\text { Research, Society And } \\
\text { Development } \\
\text { NIVEL IV }\end{array}$ & $\begin{array}{l}\text { Os autores fizeram uso da Escala de Depressão Geriátrica, composta por } 15 \text { perguntas (EDG-15) e da consulta de enfermagem } \\
\text { com anamnese de maneira direcionada. Foi feito o rastreio de depressão leve no idoso, com evidência de comprometimento nos } \\
\text { seguintes domínios dos diagnósticos de enfermagem: Domínio 1- Promoção da Saúde, Domínio 5- Percepção/Cognição, Domínio } \\
\text { 9- Enfrentamento/Tolerância ao Estresse. }\end{array}$ \\
\hline 2021 & $\begin{array}{l}\text { Rastreamento de violência contra } \\
\text { pessoas idosas: associação com } \\
\text { estresse percebido e sintomas } \\
\text { depressivos em idosos } \\
\text { hospitalizados }\end{array}$ & $\begin{array}{l}\text { Antequera } e t \\
\quad a l .\end{array}$ & $\begin{array}{l}\text { Rev. Escola Anna Nery } \\
\text { NIVEL IV }\end{array}$ & $\begin{array}{l}\text { Os enfermeiros utilizaram as Escalas de Hawlek-Sengstock Elder Abuse Screening Test (H-S/EAST) visando avaliar o risco de } \\
\text { violência no idoso, Escala de Estresse Percebido e de Depressão Geriátrica. } 44 \% \text { dos idosos apresentaram predisposição ao } \\
\text { desenvolvimento de depressão leve a severa, } 39 \% \text { com níveis elevados de estresse e } 56 \% \text { nível elevado para violação de direitos e } \\
\text { violelência. Quanto mais vulnerável mental e fisicamente o idoso estivesse, mais predisposto estaria a ocorrência de violência. }\end{array}$ \\
\hline
\end{tabular}

Fonte: Autores (2021). 


\section{Discussão}

A partir dos resultados, os autores perceberam a necessidade de agrupar a discussão em três categorias temáticas de acordo com sua representatividade durante a leitura. Categoria 1) Principais escalas para rastreio da depressão em idosos; 2) O envelhecer saudável e a depressão: solidão e resiliência na terceira idade e 3) Condutas do enfermeiro mediante o rastreio da depressão.

\subsection{Principais escalas para rastreio da depressão em idosos}

A Escala de Depressão Geriátrica (EDG) foi usada pelos enfermeiros em quase todos os estudos que compuseram esta revisão (11), demonstrando a força do instrumento para o rastreio dos sintomas depressivos na população idosa. A EDG, criada por Yesavage em 1983 é utilizada amplamente para estudos de natureza clínica, devido a sua linguagem que permite fácil aplicação além de sua confiabilidade testada em diversos outros estudos (Pereira, 2017; Ferreira, 2018; Espírito-Santo, 2018).

A escala apresenta duas versões amplamente utilizadas, uma com 30 itens (EDG-30) e outra reduzida, com 15 itens (EDG-15). Recentemente, novas propostas expõem a possibilidade de utilização da escala com apenas 5 itens, entretanto, a literatura que embasa sua confiabilidade ainda é discreta, e deste modo, a maior parte dos estudos se mantém utilizando as duas versões anteriores (Santos et al., 2019). As perguntas contidas nos instrumentos avaliam uma vasta quantidade de características que podem estar relacionadas a depressão como: a insatisfação geral com a vida, a presença de sentimentos relacionados à desamparo e/ou desesperança, sensação de medo constante em relação ao futuro, afastamento das atividades comunitárias, sensações de apatia, tédio e inutilidade diante dos acontecimentos e a experimentação de momentos recorrentes de choro, tristeza sem razão aparente e baixa autoestima geral (Pereira, 2017; Ferreira, 2018; Espírito-Santo, 2018).

Durante os estudos realizados pela Enfermagem, foi possível observar que parte dos idosos teve contato com outros diagnosticados com depressão, ou ainda apresentaram sintomatologia moderada sugestiva. A utilização da escala apontou as fragilidades mais recorrentes ao grupo, sendo que morar sozinho, não estar envolvido em atividades com outros idosos e o sedentarismo foram as mais citadas. Outro ponto importante foi a falta de conhecimento dos idosos sobre os demais sintomas depressivos, associando a doença apenas a estar sentindo-se triste, o que pode prejudicar sua autopercepção sobre a saúde (Andrade, 2016; Ferreira, 2018; Aguiar, 2016; Lentsck et al., 2015; Uchoa et al., 2019).

Apenas três outros autores utilizaram escalas diferentes da EDG. Os instrumentos Center for Epidemiologic Survey Depression, Escala abreviada de Zung e a escala Short-Care são também instrumentos validados que não estimulam o diagnóstico depressivo, apenas seu rastreio (Lentsck et al., 2015; Lima et al., 2019; Santos et al., 2020). Possuem versões traduzidas e validadas para a língua portuguesa, podendo ser usadas em estudos qualitativos ou quantitativos. Faz-se importante ressaltar a necessidade de uma maior inserção de outros instrumentos que podem ser usados pelo enfermeiro a este fim, favorecendo maior pluralidade de olhares sobre este assunto.

\subsection{O envelhecer saudável e a depressão: solidão e resiliência na terceira idade}

A senescência é um processo inevitável e fisiológico associado ao ser humano, tendo a morte como seu desfecho natural. Entretanto, quando aliado a senilidade, ou seja, ao aparecimento de doenças e/ou condições que impossibilitem o bem-estar e autonomia ao desenvolvimento de atividades da vida diária, pode tornar-se um empecilho a capacidade de enfrentamento por parte do idoso, quando ele não é acompanhado de maneira cuidadosa ao iniciar este novo ciclo (Rêgo et al., 2017; Saraiva et al., 2017).

O processo de envelhecimento foi percebido de maneira diferente pelos idosos participantes das pesquisas, tendo como um desfecho comum dentro das análises, a dificuldade em desenvolver atividades e o constante sentimento de solidão, seja pelo isolamento dentro do próprio domić́lio, ou pela mudança para Instituições de Longa Permanência (ILP). 
Dentre as ocasiões de solidão no domicilio, foi comum a observação pelos autores de sua maioria no sexo feminino. Este fato pode ser explicado tanto pela maior prevalência de mulheres viúvas, que optam por não casar novamente quando seus companheiros falecem, aliado à questão de os homens estarem mais expostos a ações de violência, falecendo mais cedo. Já as ILPs seriam locais onde, teoricamente, os idosos teriam suas necessidades contempladas e seriam acompanhados por profissionais de saúde. Entretanto, o idoso pode não se adaptar a essas situações, diminuindo sua capacidade de resiliência (Guths et al., 2017; Lima et al., 2019).

As ILP constituíram o local onde a maior parte dos estudos foram realizados. Os idosos são apresentados a estas instituições principalmente quando apresentam algum problema de saúde que demanda um maior tempo necessário ao seu lado. Foram expostas pelos idosos falas que remetem à solidão e abandono familiar, traduzidas em relatos de poucas ou nenhuma visita durante as coletas de dados. Não se sentir parte do local onde reside, ter deixado o seu lar, perder parte de sua autonomia e privacidade ou ter uma rede de apoio falha influi diretamente da percepção sobre a qualidade de vida do mesmo, favorecendo o estado deprimido (Melo et al., 2020; Nóbrega et al., 2020; Santos et al., 2020).

Uma pior qualidade de vida esteve relacionada à depressão em dois de nossos resultados. Os idosos que vivem isolados nas ILPs apresentam uma maior quantidade de domínios de qualidade de vida afetados, sendo os mais citados na literatura: autonomia, dignidade, participação social e segurança. Os reflexos psicológicos de uma qualidade de vida diminuída também repercutem fisicamente no comportamento do idoso que pode ter menor desejo em realizar tarefas simples do dia a dia, socialização diminuída, inapetência e deficiência em seu autocuidado (Melo et al., 2018; Nóbrega et al., 2020).

Os idosos devem ser estimulados pelos enfermeiros e demais cuidadores a serem parte integrante de seu autocuidado, bem como de suas tarefas, quando isto for possível. Proporcionar um maior sentimento de pertencimento, utilidade e inserção social permite ao idoso experimentar novamente sua autonomia e capacidade de enfrentamento, importante ao convívio de maneira saudável. A promoção da escuta ativa, aliada ao auxílio prestado favorecem a criação de vínculo e estimula o processo de comunicação efetiva profissional-cliente (Rêgo et al., 2017; Saraiva et al., 2017).

\subsection{Condutas do enfermeiro mediante o rastreio da depressão}

Apenas dois autores propuseram comparações ou evidenciaram a prática de enfermagem aliada aos instrumentos para rastreio da depressão (Serra et al., 2019; Silva et al., 2021a). A lacuna de estudos que proporcionem a visão do cuidado de enfermagem para além da aplicação de instrumentos, visando a incorporação do Processo de Enfermagem (PE) e Práticas Avançadas é citada por outros estudos presentes na literatura. As formas de abordagem do enfermeiro, seja na Atenção Primária (APS), hospitalar ou em Instituições de acolhimento vai além das ações apenas clínicas, e prioriza o cuidado centrado na pessoa e suas particularidades, oportunizando um amplo leque de situações a serem descritas (Lima, 2021; Silva et al., 2021b).

Serra e colaboradores (2019) evidenciam que aproximadamente 50\% menos rastreamentos de idosos depressivos foram feitos utilizando os registros dos enfermeiros em comparação aos resultados quando os mesmos utilizavam a EDG. Reafirmam o ideal de que o ser humano não pode ser compreendido apenas em sua esfera visível e a deficiência de instrumentos avaliativos complementares ao enfermeiro, que tragam a questão da depressão para a ótica do seu objeto de trabalho (Serra et al., 2019).

A Consulta de Enfermagem, procedimento regulamentado e privativo do profissional, é evidenciado como um instrumento assistencial importante para a captação das necessidades da população. O momento permite a percepção das carências clínicas, importantes ao desenvolvimento da autonomia do idoso, e sobretudo de outros determinantes que contemplam sua saúde, como o cultural, ou seja, aquilo que está envolvido em como o ser se percebe dentro da sociedade e comunidade familiar. Assim, o enfermeiro tem subsídios para enfraquecer a prática hegemônica de observação única de aspectos biológicos, quando põe em prática seu PE (Feitosa et al., 2021). 
O olhar holístico por parte do enfermeiro sob as necessidades do público idoso contribui para a diminuição dos erros diagnósticos, pois permite que informações mais precisas sejam passadas ao psicólogo dentro da equipe interprofissional. Alguns sintomas depressivos podem ser confundidos com aqueles comuns à senescência a exemplo da indisposição a algumas atividades, cansaço e mais momentos de sono. Todavia, uma anamnese direcionada e atenta que busca escutar de maneira ativa e compreender a origem dessas condições auxilia um encaminhamento mais otimizado e diminui as chances de progressão depressiva, além de viabilizar a criação de Diagnósticos de Enfermagem, que orientem suas intervenções (Lima, 2021; Silva et al., 2020).

A respeito disso, Silva et al. (2021), trouxeram a importância dos diagnósticos de enfermagem na esfera da APS para embasar o acompanhamento dos idosos. Os autores observaram alterações nos domínios de Promoção da Saúde, (auto)Percepção, Enfrentamento e Tolerância ao Estresse. A partir do entendimento dos determinantes de fragilidade foi possível elencar os riscos aos quais os idosos estavam expostos, como o de ansiedade relacionado a estressores diversos e o de memória prejudicada (Silva et al., 2021).

As condutas assumidas pelo enfermeiro deverão ter caráter equânime e longitudinal, haja visto que os clientes idosos passam por diferentes trajetórias de vida e deste modo, têm necessidades distintas. O profissional neste contexto deve agir como um encorajador da expressão de sentimentos e angústias, ação que só será possível a partir da criação de vínculo. O acolhimento tanto do idoso quanto do grupo familiar de referência, buscando entender e mediar as causas do sofrimento promoverão uma maior facilitação de entendimento sobre o problema, além de estímulo ao enfrentamento e tomada de decisão.

\section{Considerações Finais}

A partir desta pesquisa foi possível contemplar os instrumentos mais utilizados pelo enfermeiro para o rastreio da depressão em idosos a nível nacional, sendo o principal a Escala de Depressão Geriátrica.

Os enfermeiros utilizaram-se de abordagens multidimensionais, que permitiram a avaliação de outros parâmetros importantes a qualidade de vida do idoso além do estado depressivo, como a capacidade funcional, seu estado nutricional e sua capacidade de enfrentamento por meio da resiliência.

Chama-se a atenção, porém, a necessidade de criação de novos instrumentos direcionados ao objeto da Enfermagem e maior apropriação do PE, permitindo priorizar o cuidado centrado na pessoa e suas necessidades, otimizando o cuidado e assistência. Para isso, torna-se necessário o uso de campos diferentes para a realização de pesquisas futuras, bem como o emprego de outros instrumentos de rastreio dos sintomas depressivos, além da Escala de Depressão Geriátrica, para a observação e apresentação de resultados que corroborem ou permitam o desenvolvimento de novos olhares sobre a temática.

\section{Referências}

Andrade, A. B. C. A., Ferreira, A. A., \& Aguiar, M. J. G. (2016). Conhecimento dos idosos sobre os sinais e sintomas da depressão. Saúde em Redes, 2(2), 157166. https://doi.org/10.18310/2446-4813.2016v2n2p157-166

Antequera, I. G., Lopes, M. C. B. T., Batista, R. E. A., Campanharo, C. R. V., Costa, P. C. P., \& Okuno, M. F. P. (2020). Rastreamento de violência contra pessoas idosas: Associação com estresse percebido e sintomas depressivos em idosos hospitalizados. Escola Anna Nery, 25. https://doi.org/10.1590/2177-9465EAN-2020-0167

Araújo, G. K. N., Sousa, R. C. R., Souto, R. Q., Silva Júnior, E. G., Eulálio, M. do C., Alves, F. A. P., \& Neri, A. L. (2017). Capacidade funcional e depressão em idosos. Rev. enferm. UFPE on line, 3778-3786.

Cardoso, A. E. P., Rodrigues D. D., Silva, M. E. C. B., Santos, L. A. O., Lara, H. C. A. A. (2018). Prevalência de sintomas de depressão em idosos assistidos pela unidade básica de saúde. TCC-Enfermagem. Centro Universitário de Várzea Grande (UNIVAG). 13 p.

Costa, J. S. (2017). Assistência De Enfermagem Na Depressão Na Terceira Idade. 2017. 25 f. TCC - Curso de Enfermagem, Centro Universitário São Lucas, Porto Velho. 
Didoné, L. S., Jesus, I. T. M. de, Santos-Orlandi, A. A., Pavarini, S. C. I., Orlandi, F. S., Costa-Guarisco, L. P., Gratão, A. C. M., Gramany-Say, K., Cominetti, M. R., Gomes, G. A. O., \& Zazzetta, M. S. (2020). Factors associated with depressive symptoms in older adults in context of social vulnerability. Revista Brasileira de Enfermagem, 73. https://doi.org/10.1590/0034-7167-2019-0107

Feitosa, J. P., Silva, M. A. B., Lima, J. G., \& Vieira, R. P. (2021). Percepções de Enfermeiros acerca da Depressão em Idosos / Nurses’ Perceptions of Depression in the Elderly. ID on line. Revista de psicologia, 15(55), 553-574. https://doi.org/10.14295/idonline.v15i55.3092

Ferreira, C. F. D., \& Espirito-Santo, H. (Orientadora). (2018). Validação da Escala de Depressão Geriátrica numa Amostra de Idosos Institucionalizados da População Portuguesa. http://repositorio.ismt.pt/jspui/handle/123456789/931

Guimarães, L. A., Brito, T. A., Pithon, K. R., Jesus, C. S., Souto, C. S., Souza, S. J. N., \& Santos, T. S. (2019). Sintomas depressivos e fatores associados em idosos residentes em instituições de longa permanência. Ciência \& Saúde Coletiva, 24, 3275-3282. https://doi.org/10.1590/1413-81232018249.30942017

Güths, J. F. S., Jacob, M. H. V. M., Santos, A. M. P. V., Arossi, G. A., \& Béria, J. U. (2017). Sociodemographic profile, family aspects, perception of health, functional capacity and depression in institutionalized elderly persons from the north coastal region of Rio Grande do Sul, Brazil. Revista Brasileira de Geriatria e Gerontologia, 20, 175-185. https://doi.org/10.1590/1981-22562017020.160058

Lentsck, M. H., Pilger, C., Schoereder, E. P., Prezotto, K. H., \& Mathias, T. A. F. (2015). Prevalência de sintomas depressivos e sinais de demência em idosos na comunidade. Revista Eletrônica de Enfermagem, 17(3), Article 3. https://doi.org/10.5216/ree.v17i3.34261

Lima, A. M. P., Ramos, J. L. S., Bezerra, I. M. P., Rocha, R. P. B., Batista, H. M. T., \& Pinheiro, W. R. (2016). Depressão em idosos: Uma revisão sistemática da literatura. Revista de Epidemiologia e Controle de Infecção, 6(2), 96-103. https://doi.org/10.17058/reci.v6i2.6427

Lima, G. S., Souza, I. M. O., Storti, L. B., Silva, M. M. J., Kusumota, L., \& Marques, S. (2019). Resiliência, qualidade de vida e sintomas depressivos entre idosos em tratamento ambulatorial. Revista Latino-Americana de Enfermagem, 27. https://doi.org/10.1590/1518-8345.3133.3212

Lima, Y. B. R. S., Miranda A. C., Jucá, E. S., Dias, S. C. V., Pinheiro, S. K. L., Silva, S. S. S., Viana, A. P. M. R., Portilho, P. G. M., Martins, M. B., \& Aguiar, V. F. F. (2021). A enfermagem frente às manifestações clínicas da depressão em idosos que sofreram abandono: revisão integrativa da literatura. Revista Eletrônica Acervo Saúde, 13(2), e4162. https://doi.org/10.25248/reas.e4162.2021

Melo, L. A., Jesus, I. T. M., Orlandi, F. S., Gomes, G. A. O., Zazzetta, M. S., Brito, T. R. P., \& Santos-Orlandi, A. A. (2020). Fragilidade, depressão e qualidade de vida: Um estudo com idosos cuidadores. Revista Brasileira de Enfermagem, 73. https://doi.org/10.1590/0034-7167-2018-0947

Melo, L. A., Andrade, L., Silva, H. R. O., Zazzetta, M. S., Santos-Orlandi, A. A., \& Orlandi, F. S. (2018). Fragilidade, sintomas depressivos e qualidade de vida: um estudo com idosos institucionalizados. Revista Baiana de Enfermagem32, . https://doi.org/10.18471/rbe.v32.26340

Nóbrega, I. S., Medeiros, T. P. G., Marcolino, E. C., Santos, R. C., Souto, R. Q., \& Andrade, L. T. (2020). Avaliação da depressão e da qualidade de vida de idosos institucionalizados. Revista Enfermagem Atual In Derme, 94(32), e-020077. https://doi.org/10.31011/reaid-2020-v.94-n.32-art.879

Pereira, K. R. Adaptação Transcultural e Validação Da Escala De Depressão Geriátrica Gds-15 (2017). Dissertação (Mestrado. Programa de Pós-Graduação Stricto Sensu em Atenção À Saúde, Universidade Federal do Triângulo Mineiro, Uberaba. 67 f.

Pereira, M. M. V., Rufino, M. H. de O., Nascimento, L. C., Macêdo, R. da C., Oliveira, R. K. C. de, \& Freire, J. A. P. (2015). Depressão e estado nutricional de idosos participantes do Programa Hiperdia. Rev Rene (Online), 731-737.

Ramos, F. P., Silva, S. C., Freitas, D. F., Gangussu, L. M. B., Bicalho, A. H., Sousa, B. V. O., Rametta, Z. M. J., Rametta, F. J., Rametta, F. J., Rametta, L. P. M., Nascimento, C. I. C., Santos, S. H. S., \& Guimarães, T. A. (2019). Fatores associados à depressão em idoso. Revista Eletrônica Acervo Saúde, (19), e239. https://doi.org/10.25248/reas.e239.2019

Rêgo, R., Jesus, I. M., Cruz, G. H. S., Souza, M. S., Amaral, E. O., \& Teles, M. A. B. (2017). Assistência prestada por Enfermeiros da Estratégia de Saúde da Família à população idosa do município de Montes Claros-MG. Almanaque Multidisciplinar de Pesquisa, 4(2), Article 2. http://publicacoes.unigranrio.edu.br/index.php/amp/article/view/4385

Santos, A. J., Nunes, B., Kislaya, I., Gil, A. P., \& Ribeiro, O. (2019). Estudo de validação em Portugal de uma versão reduzida da escala de depressão geriátrica. Análise Psicológica, 37(3), 405-415. https://doi.org/10.14417/ap.1505

Santos, J. O., Aguiar, B. F., Tonin, L., \& Rozin, L. (2020). Autoestima e risco para depressão em idosos residentes em instituições de longa permanência. Espaço para a Saúde, 21(1), 59-70. https://doi.org/10.22421/15177130-2020v21n1p59

Saraiva, L. B., Santos, S. N. S. A. dos, Oliveira, F. A., Almeida, A. N. S., Moura, D. J., \& Barbosa, R. G. B. (2017). Avaliação Geriátrica Ampla e sua Utilização no Cuidado de Enfermagem a Pessoas Idosas. Journal of Health Sciences, 19(4), 262-267. https://doi.org/10.17921/2447-8938.2017v19n4p262-267

Serra, M. A., Nascimento, I. M. T., Guimarães, G. L., Silva, S. M., Goveia, V. R., \& Mendoza, I. Y. Q. (2019). Prevalência de sintomas depressivos no idoso hospitalizado: Estudo comparativo. Rev. enferm. UERJ, e36091-e36091.

Silva, B., Anjos, I., Neto, G., Santana, D., Araújo, J., Alves, D., Lima, J., Santos, A., Araújo, M., Nascimento, M., Batista, A., Macedo, L., Furtado, A., \& Aguiar, V. (2021). Importância da identificação do diagnóstico de enfermagem ao paciente com depressão senil na atenção básica. Research, Society and Development, 10, e53510212770. https://doi.org/10.33448/rsd-v10i2.12770

Silva, C. J. A., Cassiano, A. N., Lima, M. C. R. A. A., Peruhype, R. C., Queiroz, A. A. R., \& Menezes, R. M. P. . (2021). Perspectivas da Prática Avançada de Enfermagem no processo de cuidado gerontológico: Revisão integrativa. Revista Eletrônica de Enfermagem, 23. https://doi.org/10.5216/ree.v23.68003

Silva, V. PO., Carneiro, L. V., Lucena, W. M. A., Alixandre, A. L., \& Oliveira, J. S. (2020). Escala de depressão geriátrica como instrumento assistencial do enfermeiro no rastreio de sintomas depressivos em idosos institucionalizados / Geriatric depression scale as a nurse's assistance instrument in the screening of depressive symptoms in institutionalized elderly people. Brazilian Journal of Development, 6(3), 12166-12177. https://doi.org/10.34117/bjdv6n3-188 
Research, Society and Development, v. 11, n. 1, e13811124566, 2022

(CC BY 4.0) | ISSN 2525-3409 | DOI: http://dx.doi.org/10.33448/rsd-v11i1.24566

Sousa, L. M. M. D., Firmino, C. F., Marques-Vieira, C. M. A., Severino, S. S. P., \& Pestana, H. C. F. C. (2018). Revisões da Literatura Científica: tipos, métodos e aplicações em enfermagem. Revista Portuguesa de Enfermagem de Reabilitação, 1(1), 45-54. https://doi.org/10.33194/rper.2018.v1.n1.07.4391

Stillwell, S. B., Fineout-Overholt, E., Melnyk, BM., \& Williamson, K. M. (2010). Evidence-based practice, step by step: Asking the clinical question: a key step in evidence-based practice. The American Journal of Nursing, 110(3), 58-61. https://doi.org/10.1097/01.NAJ.0000368959.11129.79

Uchoa, V. S., Chaves, L. L., Botelho, E. P., Polaro, S. H. I., \& Oliveira, M. F. V. (2019). Fatores Associados a Sintomas Depressivos e Capacidade Funcional em Idosos. Cogitare Enfermagem, 24(0), Article 0. https://doi.org/10.5380/ce.v24i0.60868

Wanderley, R. M. M., Cunha, D. G. P., Felisberto, A. M. S., Oliveira, B. R. S., Bittencourt, G. K. G. D., Amaral, A. K. F. J., \& Silva, A. O. (2019). Avaliação da condição de saúde da pessoa idosa na atenção básica. Rev. enferm. UFPE on line, 472-482. 